\title{
Temporal and spatial variations of global ionospheric total electron content under various solar conditions
}

\author{
Jingbin Liu¹, Manuel Hernandez-Pajares², Xinlian Liang, Juha Hyyppä1, Ruizhi Chen \\ 1. Department of Remote Sensing and Photogrammetry, Finnish Geodetic Institute, Finland \\ 2. Department of Applied Mathematics IV, Technical University of Catalonia, Spain \\ 3. Conrad Blucher Institute for Surveying \& Science, Texas A\&M University Corpus Christi, USA
}

\section{Key points}

- Spatial and temporal variability of the ionosphere for a solar cycle and different local times

- The PCA discloses periodic variations as well the spatiotemporal asymmetries

- Newly found trend and characteristic epochs of the variability with respect to local times

\begin{abstract}
By utilizing the numerical technique of principal component analysis (PCA), this work investigated temporal and spatial variations of the ionosphere under the various solar conditions. Global ionospheric total electron content (TEC) maps of 1999-2013 derived from Global Navigation Satellite Systems (GNSS) used in this work were reorganized according to local time (LT). Applying the PCA technique to the time series of global TEC maps provides an efficient method to analyze the main ionospheric variability on a global scale, and it is able to decompose periodic variations (e.g. annual and semiannual oscillations), as well retain the asymmetry in temporal and spatial domains (e.g. seasonal and equator anomalies). The TEC series of different local times are processed separately in two time scales: the whole 15 years of the period of study and individual years. Different from previous studies, the analysis on the dataset of the 15 years showed that dawn time (e.g. LT4:00) and later morning (LT10:00-12:00) are more remarkable characteristic epochs for the ionosphere variability. This study showed also a cyclic trend of the variability with respect to local times. The first two modes, which contain $80 \%-90 \%$ of the total variance, represent spatial distributions and temporal variations with respect to different characteristic stages of a solar cycle and local times. Annual and semiannual variations are demodulated from the first two modes, and the results showed that these oscillation variations have evidently different patterns for daytime and nighttime. An exception is that, under active solar conditions, extremely strong solar irradiance of daytime has remaining effect on the variability of the ionosphere even after the sunset.
\end{abstract}

Index terms: Ionospheric physics; Solar radiation and cosmic ray effects; Modeling and forecasting; Solar activity cycle; Arctic region

Key Words: Global ionosphere; Principal component analysis; Total electron content; Ionosphere TEC mapping; Arctic navigation

\section{Introduction}

The Earth's ionosphere ionized mainly solar and cosmic radiation has complicated processes of dynamics, spatial and temporal variations of which reflect the behavior of electron concentration in 
the ionosphere and indicate the state of the ionospheric plasma. It is important for us to understand the state of the ionosphere because it influences. Total electron content (TEC) is one of the physical parameters indicating the state of the ionosphere. The development of methods for studying and modeling the dynamics of TEC is stipulated not only by a purely scientific interest to the research of the upper atmosphere of the Earth, but also by the necessity to solve some applied problems concerning radio propagation around the Earth and between satellites and the Earth, based on which many technical systems are, such as radio communication, satellite positioning and radiolocation systems.

Chapman [1931] developed a formula that describes the rate of production of ion-electron pairs as the product of four terms: the intensity of ionizing radiation at some level of the atmosphere, the concentration of atoms or molecules capable of being ionized by that radiation, the absorption cross section, and the ionization efficiency. Any departure from this "solar-controlled" behavior in the ionosphere is considered as anomaly. Several authors have analyzed various variations and have identified three major components: winter maximum (seasonal anomaly), equinoctial maxima (semiannual anomaly), and annual anomaly, which are analyzed using using F2-layer peak electron content (NmF2) and TEC (Rishbeth, 1998; Rishbeth et al., 2000; Zou et al., 2000; Liu et al., 2009; Zhao et al., 2005; Meza and Natali, 2008). Winter or seasonal anomaly: noon values of $N m \mathrm{~F} 2$ are greater in winter than in summer, whereas the Chapman theory leads us to expect the opposite. The anomaly disappears at night. The winter anomaly falls off in amplitude and area with decreasing solar activity. Semiannual anomaly: $N m F 2$ is abnormally large at equinoxes. The semiannual variation appears around the globe at daytime, and except in low latitude region and in South American sector ( $\mathrm{Li}$ and $\mathrm{Yu}, 2003$ ) there is no obvious semiannual variation of $N m \mathrm{~F} 2$ in the nighttime. The amplitude of this variation has close relationship with the solar activity, they have asymmetrical structures between the two hemispheres (larger at the Northern Hemisphere) and they have longitude difference (Ma et al., 2003). Annual anomaly: in the world as a whole December $N m \mathrm{~F} 2$ is on average greater than June $N m \mathrm{~F} 2$, both by day and by night. At low solar activity, there are regions of summer maximum (i.e. no anomaly) at equatorial and southern latitudes. Many theoretical interpretations of these phenomena have been proposed. Among the most accepted theories we can mention: Torr and Torr [1973] analyzed $N m \mathrm{~F} 2$ variations in winter maximum, equinoctial maxima and a component which peaks in December-January, and they constructed global maps that represent the anomalies for different solar activity, and Rishbeth and Setty [1961] proposed that the winter or seasonal anomaly could be attributed to a seasonal change in chemical composition of the neutral air. Several authors have found different explanations to the semiannual anomaly, like variations in the solar wind [Lal, 1992, 1998] or an increase of the internal thermospheric mixing at solstice [Fuller - Rowell, 1998]. A possible cause of the annual anomaly is the changing Earth - Sun distance between June and December together with some associated atmospheric processes [Buonsanto, 1986]. The solar-cycle changes in the domains of the annual and semiannual effects have yet to be explained, but may be connected with changes in the strengths of the global circulation. One of difficulties in capturing and explaining the variability of the ionosphere is due to that traditional observation techniques have limited samples in temporal and spatial domains.

The development of Global Navigation Satellite Systems (GNSS) has resulted in a global observation network that provides data for studying the global dynamics of the ionosphere with unprecedented spatial and time resolution. An increasing number of GNSS receivers coordinated by the International GNSS Services (IGS) have distributed over the global during the past two decades to continuously acquire measurements (reference ???). In May 1998, IGS created the Ionosphere Working Group to generate routinely ionospheric vertical (TEC) maps [Feltens and Schaer, 1998]. Of the scientific groups and institutes that are presently dedicated to ionospheric studies using GPS 
observations, three can be distinguished as the most important: the Center for Orbit Determination in Europe (CODE) at the Astronomical Institute of the University of Bern, Switzerland (http://www.aiub.unibe.ch/igs.html); the Astronomy and Geomatics group (gAGE) (http://gage1.upc.es), Barcelona, Spain; and the NASA Jet Propulsion Laboratory (JPL) (http:// iono.jpl.nasa.gov), Pasadena, California. Global Ionosphere Maps (GIMs), which contain global TEC information and associated RMS, are produced by the IGS associate analysis centers, and distributed globally in a common IONosphere map EXchange (IONEX) format [Schaer et al., 1998]. Spatial resolution of vertical TEC in IONEX files is $2.5^{\circ}$ in latitude and $5^{\circ}$ in longitude, and temporal resolution is 2 hours. The long-term archive of GIM data since May 1998 has allowed one to analyzing the variability of the ionosphere in high temporal and spatial resolutions at the global scale.

This paper analyzes the dynamics and variability of the ionosphere in temporal and spatial scales using the GIM TEC archive and the Principal Component Analysis (PCA) technique. The PCA, which is also called the Empirical Orthogonal Functions (EOF), is the decomposition of a data set into a base of orthonormal functions that are directly determined by the data set itself. The PCA method has been used for a long time for many problems connected with the analysis of multivariate time series, e.g. the analysis of spatial or temporal variability of physical fields by meteorologists and oceanographers, because it is able to identify spatial structures that have dominant contribution to the total variability together with their time evolution without the need to propose any particular a priori functional model. It was recently proven that the PCA is an efficient tool for analyzing the annual, semiannual, and seasonal effects in the global TEC [Natali and Meza 2011]. It was showed that the obtained components of the PCA decomposition essentially depend on the representation of original dataset, such as data centering and coordinate systems [Maslennikova et al., 2014]. However, these works only utilized a limited dataset of the assumed time epochs (e.g. LT 1200 \& 2200) and a short time window (e.g. one year).

Applying the PCA technique on time series of GIM TEC provides us an efficient tool to analyze the main ionosphere variability on a global scale. This study analyzes temporal and spatial variations of the ionosphere for different local times throughout the period of more than a solar cycle (1999-2013) using the global GIM TEC dataset. After a brief description of the PCA method and data processing, the PCA method is applied to the TEC dataset of two different time scales: 15 years as a whole and each of individual years. Then the analysis on the results is described. Particular characteristics associated with different local times and with the geomagnetic region are highlighted. Finally, the ionosphere variability throughout the various solar conditions is discussed.

\section{Methodology}

\subsection{Principal Component Analysis}

The PCA transforms a set of correlated variables into a number of uncorrelated variables, which are based on a new orthonormal base of minimum dimension. This method was proposed by K. Pearson in 1901 (Pearson, 1901); in the sequel it was repeatedly developed further. From the mathematical point of view, the orthonormal base of the decomposition consists of eigenvectors of the variancecovariance matrix formed of initial data set. The PCA method determines the shape of the base functions from the data set itself, and it hence is more interesting than other techniques (e.g. Fourier analysis) when the phenomena under study are not necessarily a superposition of determinate function components. Within recent 20 years it became popular for identifying the principal modes of various spatial or temporal variability of physical fields of the atmosphere and ionosphere [Nerem et al., 1994, 1997; Meza and Natali, 2010, 2011; Maslennikova et. al., 2014]. The algebraic 
essentials of PCA is presented briefly in this section, and more details are referred to Preisendorfer [1988] and Storch and Zwiers [1999], for example.

Let us represent the data set of the global TEC as a matrix $X=\{X(p, t)\}_{M \times N}$, where the subscript $p$ indicates a grid point of GIM, while the subscript $t$ does the corresponding epoch time, $M$ is the length of the time series, and $N$ is the number of observation points of an epoch. As depicted in the following subsection, the GIM TEC dataset in the IONEX format is reorganized according to local time of reference, and TEC values of different local times are grouped as individual matrices $X$, and each of these matrices is processed separately.

As the first step of the PCA, a centering operation is performed so that the temporal means of TEC values of each grid point $p$ are removed from the time series:

$\check{X}=\{\check{X}(p, t)\}_{M \times N}=\left\{X(p, t)-\frac{1}{M} \sum X(p)\right\}_{M \times N}$

where $\check{X}$ is the centered data set.

Generally, the idea of PCA is to represent the original centered data set $\check{X}$ within a set of orthogonal bases as follows:

$\check{X}(p, t)=\sum_{k=1}^{N} a_{k}(t) e_{k}(p)$

where $e_{k}(p), k=1, \cdots, N$ is a set of orthogonal base of decomposition, and $a_{k}(t)$ is the principal component (or time series/function) of the corresponding base function $e_{k}$.

The orthogonal base functions and associated principal components are empirically resolved from the variance-covariance matrix of the dataset $\check{X}$, which is also called the scatter matrix (Preisendorfer, 1988), and is constructed as follows:

$S\left(p, p^{\prime}\right)=\sum_{t} \check{X}(p, t) \check{X}\left(p^{\prime}, t\right)$

where $S\left(p, p^{\prime}\right)$ is the scatter matrix of the data set $\check{X}$.

Since the covariance matrix $S\left(p, p^{\prime}\right)$ is real-valued and symmetric, it has real eigenvalues and eigenvectors, the number of which is $N$. The most important property of the eigenvectors is that they are mutually uncorrelated over dimensions, as mathematically expressed in Eq. (4).

$\sum_{p=1}^{N} e_{j}^{T}(p) e_{k}(p)=\left\{\begin{array}{ll}1 & j=k \\ 0 & j \neq k\end{array} \quad j, k=1,2, \cdots N\right.$

The eigenvectors form a set of base functions for the expansion in Eq. (2), and the corresponding eigenvalues $q_{j}(j=1, \cdots, N)$ give the amount of variance in the original dataset that is accounted for by the associated eigenvector and its time function. The eigenvalues are sorted into decreasing order, and they are normalized to have the unit summation $\left(\sum q_{j}=1\right)$. The associated principal components $a_{k}(t)$ are the decomposition time function of original dataset in the dimension of a corresponding base function $\left(e_{k}\right)$. They are computed from the original data and the eigenvectors as:

$a_{k}(t)=\sum_{p=1}^{N} \check{X}(p, t) e_{k}(p)$ 
The principal components $a_{k}(t)$ are allowed to carry the variance of the original data set, and they are also mutually orthogonal as follows:

$\sum_{t=1}^{M} a_{j}^{T}(t) a_{k}(t)=\left\{\begin{array}{cc}q_{j} & j=k \\ 0 & j \neq k\end{array} \quad j, k=1,2, \cdots N\right.$

where $q_{j}$ is the $\mathrm{j}$-th eigenvalue of the scatter matrix $S$.

Therefore, the set of eigenvectors are empirical orthogonal functions: "empirical" indicates they arise from data itself, while the orthogonality means that these base functions and principal components are uncorrelated over dimensions. This fact facilitates the study of contributions of various factors to the variability of TEC. The eigenvalues indicate the degrees of variances in the corresponding dimensions. The PCA decomposition identifies the spatial structures of the ionosphere variability that have dominant contributions to the total variance (given by the sum of all eigenvalues). The spatial structure of the ionosphere variability is represented by the eigenvector and its temporal evolution is described by time functions $a_{k}(t)$, called principal components. Eigenvector and principal components together are called mode. Modes are ordered according to decreasing eigenvalues, such that the first mode represents the largest part of the variance, the next mode the second largest part, etc.

When interpreting the data of the decomposition modes, it is necessary to take into account the fact that just the correlation of signs at two points of a map is essential, rather than signs of variations themselves. Really, one should multiply the obtained distribution by the time dependent amplitude. In the decomposition of centered data, the mentioned amplitude may have any sign. But if signs of variations of a given mode at two points coincide (or are opposite), then this means that some process connected with this principal component leads to correlating (anticorrelating, respectively) variations at these points.

\subsection{Material and data processing}

The Global Ionosphere Maps generated by the IGS analysis centers are distributed to the users in the common IONosphere Map Exchange (IONEX) format (Schaer et al., 1998). The IONEX format allows the exchange of global electron density and associated RMS referring to predefined reference epochs and to a 2D or even 3D Earth-fixed grid. In this work, we use GIM TEC dataset of 1999 - 2013 provided by CODE in the IONEX format. These global ionosphere maps show a series of snapshots of global ionospheric TEC at reference epochs of every 2 hours in the UT (Universal Time) scale, and in spatial they present TEC values and associated RMS in the form of a grid of 2.5 degrees in latitude and 5 degrees in longitude. The grid ranges from $-180^{\circ}$ to $180^{\circ}$ in longitude and from $-87.5^{\circ}$ to $87.5^{\circ}$ in latitude.

In the PCA method, in order to perform correctly the analysis of the dynamics of the series under consideration, it is necessary to take into account the specificity of the data representation and the known time trends. The TEC maps analyzed in this study have significant ionization variation due to solar radiation, which is related to the Sun's hour angle or Local time of the grid points. For example, the works (Wan et al., 2012 and Zhang Ercha et al., 2012) analyzed the dynamics of global TEC represented in local time using the EOF methods. As a result, the first mode of the decomposition with the maximal energy reflected only the known properties, such as diurnal variation of TEC, of the dynamics of the ionosphere. In this study, we filter out the well-known and extremely dominative diurnal variation by reorganizing the global TEC maps. The original reference epochs in UT of TEC maps are transformed to Local Time (LT) of the grid points, and 
these TEC values are re-grouped into twelve sets of TEC maps according to local time. The reorganized twelve sets of TEC maps have the reference epochs of every two hours local time, such as LT 0:00, 2:00, 4:00, .., 22:00, respectively. In spatial, the grid points remain as the same as the original GIMs. The interpolation methods in (Schaer et al., 1998) are utilized in the process of reorganization. Consequently, we construct the global TEC maps of every two hours local time during the 15-year interval 1999-2013. Finally, the PCA is applied to each of the twelve global TEC series of different local times separately, and the results are analyzed in the following section.

\section{Results and discussion}

Applying the PCA decomposition to the twelve sets of TEC maps provides insight into ionospheric behavior of 24 hours local time for the period of more than one solar cycle that covers various solar activity conditions. The principal components $\left(a_{k}(t)\right)$ and structural dimensions $\left(e_{k}(p)\right)$ associated to specific data sets represent the variability of the ionosphere in temporal and spatial scales. As the geophysical references of solar activity conditions during the period from 1999 to 2013, Sunspot numbers and solar radio flux F10.7 data are showed in Fig.1, which showed that the study period includes the recent extremely prolonged solar minimum (2007-2009) and active solar conditions (2000-2003 and 2012-2013). In section 3.1, we analyze TEC variations of different local times obtained from the whole 15-year dataset, and then in section 3.2 we study TEC variations year by year under different solar activity conditions.
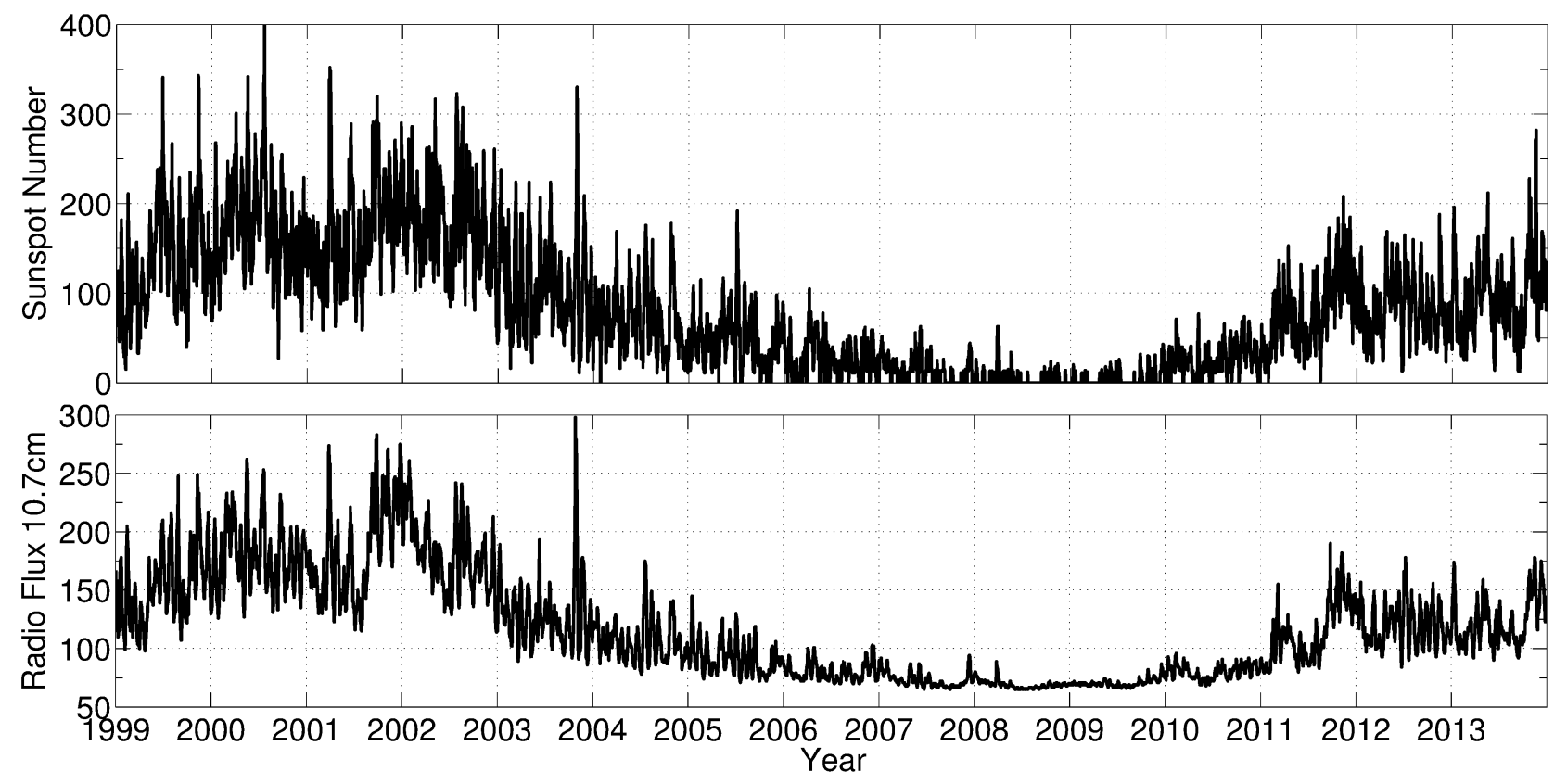

Fig. 1 Solar activity indicators of the period 1999-2013: Sunspot numbers (top) and solar radio flux F10.7 index (bottom).

\subsection{Analysis using the whole 15-year TEC dataset}

The twelve TEC series of the whole 15-year period, which are referred to every two hours local times, are processed separately with the PCA. Normalized variances for the first four modes of the decomposition are presented in Table 1 and Fig. 2. It was showed that these variances are highly dependent of local times, and the most featured phases are dawn time (LT 4:00) and later morning to noon time (LT 10:00-12:00). The first two modes account for more than 80\% (dawn time) - 90\% (LT 10:00-12:00) variations. The first mode has the maximum contribution (90\%) at LT 10:00, while the second mode becomes the most significant (26\%) at dawn time (LT 4:00). A diurnal 
cyclic trend of the first mode is that: the first mode gets the peak (90\%) at LT 10:00-12:00, and it then gets slowly down to $85.6 \%$ in the afternoon. It jumps down to $76 \%$ at dusk time (LT 18:00), and reduces gradually down to $55 \%$ at dawn time of next day. As the Sun rises up, the contribution of the first mode goes up quickly, and comes to the peak at LT 10:00. With the similar timeline, the second mode has an opposite trend in quantity. That is, the second mode has the lowest variance at noon time, and its variance gets higher in the afternoon and the nighttime. At dawn time, the second mode has the largest variance. After then, the second mode becomes weaker quickly until noon time.

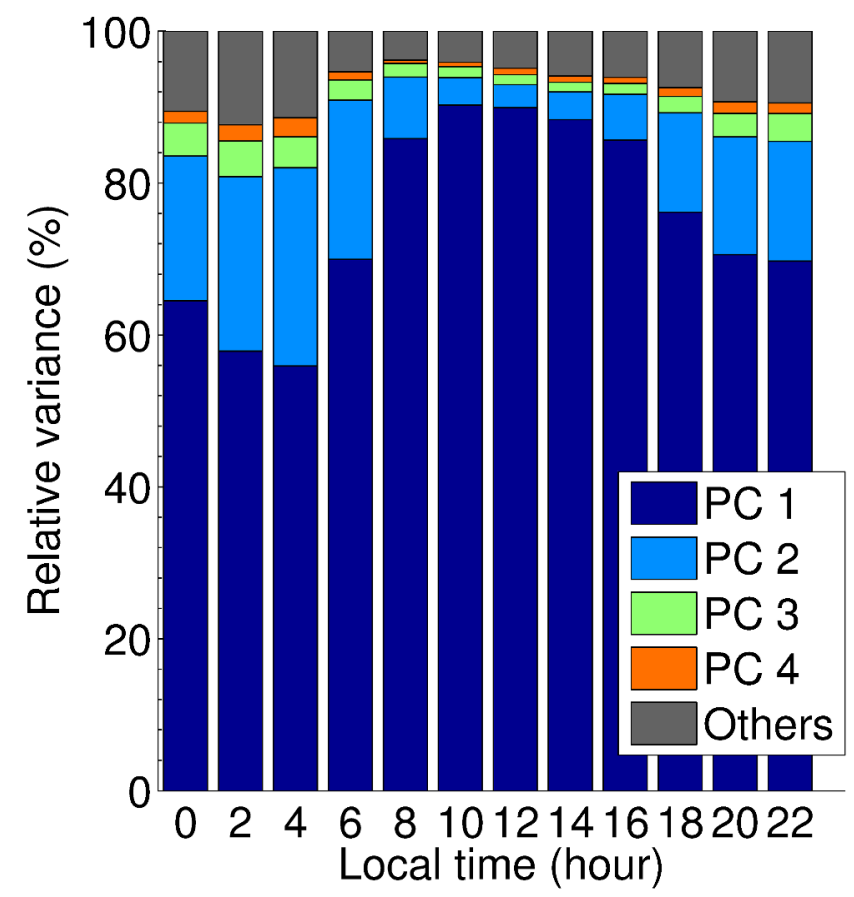

Fig. 2 Normalized variances of the first four principal components of the 12 sections, each of which covers 2 hours of local time.

Table 1. Normalized contributions of the first four components to the total variance of TEC

\begin{tabular}{c|c|c|c|c||c|c|c|c|c}
\hline \multirow{2}{*}{$\begin{array}{l}\text { Reference } \\
\text { local time }\end{array}$} & \multicolumn{9}{|c||}{ Integral value of the relative variance (\%) } \\
\cline { 2 - 10 } & PC1 & PC2 & PC3 & PC4 & PC1 & PC2 & PC3 & PC4 & Others \\
\hline 0 & 64.44 & 19.23 & 4.30 & 1.45 & 64.44 & 83.67 & 87.97 & 89.42 & 10.58 \\
\hline 2 & 57.80 & 23.14 & 4.67 & 2.01 & 57.80 & 80.94 & 85.61 & 87.62 & 12.38 \\
\hline 4 & 55.85 & 26.11 & 4.06 & 2.41 & 55.85 & 81.96 & 86.02 & 88.43 & 11.57 \\
\hline 6 & 69.94 & 21.00 & 2.63 & 1.05 & 69.94 & 90.94 & 93.57 & 94.62 & 5.38 \\
\hline 8 & 85.83 & 8.12 & 1.80 & 0.42 & 85.83 & 93.95 & 95.75 & 96.17 & 3.83 \\
\hline 10 & 90.27 & 3.62 & 1.42 & 0.62 & 90.27 & 93.89 & 95.31 & 95.93 & 4.07 \\
\hline 12 & 89.81 & 3.04 & 1.28 & 0.90 & 89.81 & 92.85 & 94.13 & 95.03 & 4.97 \\
\hline 14 & 88.29 & 3.64 & 1.31 & 0.77 & 88.29 & 91.93 & 93.24 & 94.01 & 5.99 \\
\hline 16 & 85.62 & 6.02 & 1.44 & 0.80 & 85.62 & 91.64 & 93.08 & 93.88 & 6.12 \\
\hline 18 & 76.07 & 13.12 & 2.17 & 1.14 & 76.07 & 89.19 & 91.36 & 92.50 & 7.50 \\
\hline 20 & 70.78 & 15.49 & 3.05 & 1.44 & 70.78 & 86.27 & 89.32 & 90.76 & 9.24 \\
\hline 22 & 69.80 & 15.79 & 3.58 & 1.36 & 69.80 & 85.59 & 89.17 & 90.53 & 9.47 \\
\hline
\end{tabular}

The diurnal cyclic trend of the first two modes has two characteristic epochs of LT 4:00 and 10:00. Fig. 3 showed the time series and spatial distribution of the first mode of LT 4:00 and 10:00, respectively. The principal component of mode 1 has a main annual oscillation component 
modulated by a semiannual oscillation component. These two oscillation components have timevarying amplitudes. In order to demodulate these two components, the Fourier analysis as follows together with a sliding window of one-year length is applied to the principal component of the first mode at the two characteristic epochs.

$$
\begin{aligned}
\psi(t) & =C_{0}+\sum_{i=1}^{N}\left[C_{i} \cos \left(w_{i} t\right)+S_{i} \sin \left(w_{i} t\right)\right] \\
& =C_{0}+\sum_{i=1}^{N}\left[A_{i} \cos \left(w_{i} t-\phi_{i}\right)\right] \quad w_{i}=2 \pi / p_{i}, A_{i}=\sqrt{C_{i}^{2}+S_{i}^{2}}
\end{aligned}
$$

where $\psi(t)$ is a time series of the sliding window, $C_{0}$ is the average of the time series in the sliding window, $N$ is the number of components ( $N=2$ for annual and semiannual components), $w_{i}$ is the angular frequency of a component with a period $p_{i}$, and $C_{i}$ and $S_{i}$ are coefficients to be estimated, which define the phase $\left(A_{i}\right)$ and amplitude $\left(\phi_{i}\right)$ of the corresponding components.

As showed in Fig. 4, time-varying amplitudes of annual and semiannual oscillation components in the first mode are highly dependent of local times and solar activities. At the both epochs, the amplitudes of these two components under active solar activity (e.g. 2001-2002) might be up to six times higher than that of calm solar condition (2007-2009). At dawn time, the amplitude of annual oscillation is roughly twice that of semiannual oscillation under the various solar activities, whereas at noon time the amplitude of annual oscillation is comparable with that of semiannual oscillation during most of the whole period. At dawn time, the both components have no significant phase shift, whereas they have 180 degrees phase shift for each other at noon time.

In spatial, for each of the two epochs, the eigenvector of the first mode has the same sign at the global, which means that the first mode is correlating globally. However, the spatial distributions of the first mode of the two epochs are significantly different, as showed in Fig. 3. At dawn time, geomagnetic Southern hemisphere has higher variance than Northern hemisphere. The region between South American and the Antarctic has the highest variance of the first mode, and its symmetric area with respect to the geomagnetic equator, i.e. Southern Europe and Middle East region in Northern hemisphere, has the lowest variance. At LT 10:00, the spatial distribution of the first mode is highly dependent of geomagnetic latitudes, and it is symmetric with respect to the geomagnetic equatorial. The most characteristic feature of the spatial distribution at LT 10:00 is that the maximum variation is distributed within the band from the geomagnetic equator (GE) to $\pm 20^{\circ}$ geomagnetic latitudes (ML), which corresponds to the area where equatorial anomaly occurs. The degree of this variation decreases with increasing ML. 


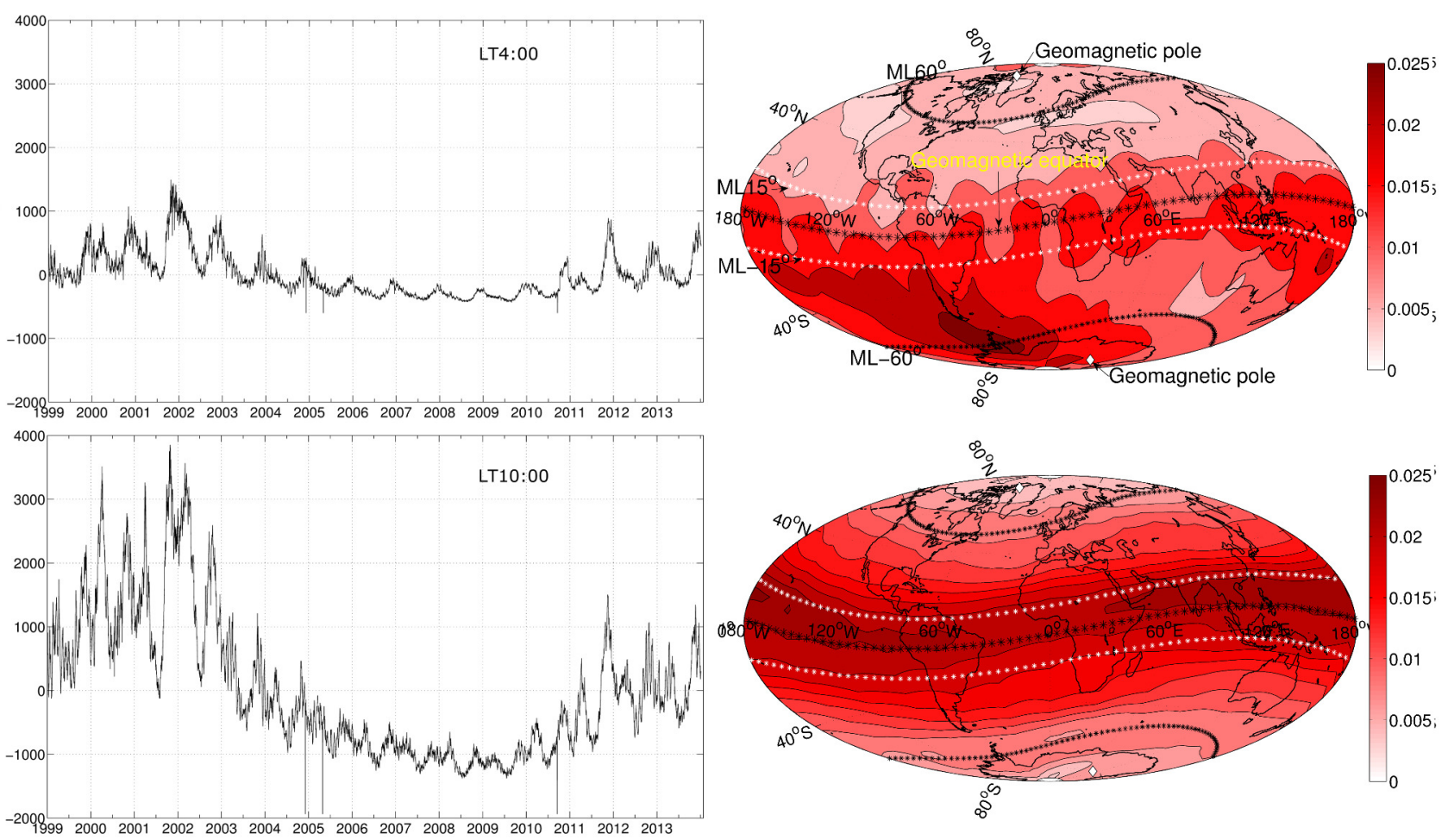

Fig. 3 Temporal and spatial variations in the first mode of the ionosphere of 1999-2013 for local times: LT 4:00 (upper) and LT10:00 (lower). In the subplots of spatial variation, southern and northern geomagnetic poles are marked by a while diamond respectively, geomagnetic equator and southern and northern ML $60^{\circ}$ are marked by a black-star line respectively, southern and northern ML $15^{\circ}$ are marked by a white-star line respectively.

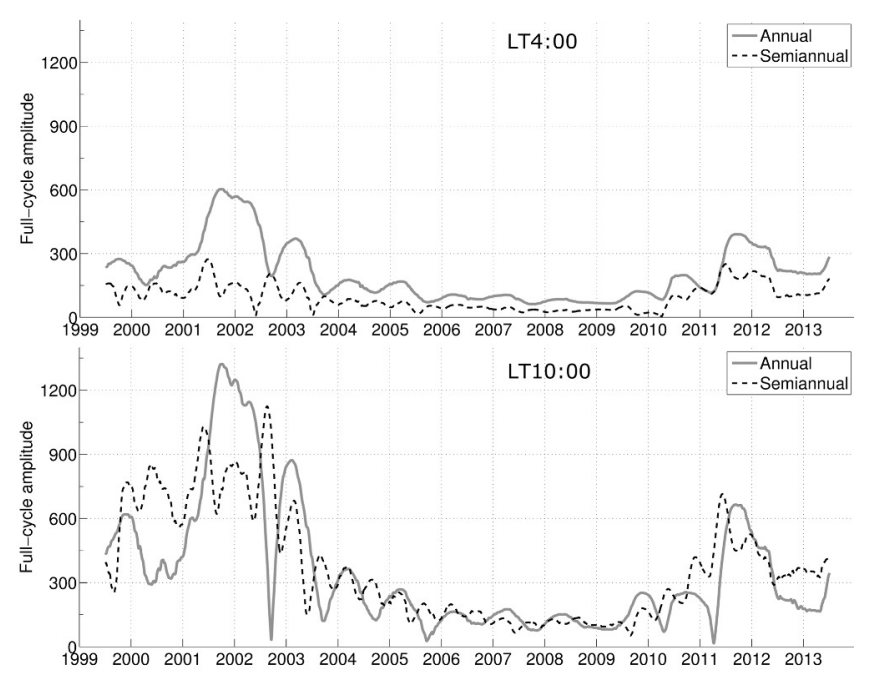

Fig. 4 The amplitudes of annual and semiannual components in the temporal variation of the first mode for local time LT 4:00 and LT 10:00, respectively.

As to the second mode, the spatial structure of the variation is symmetric with respect to the geomagnetic equator, and the variation is anticorrelating at Southern and Northern hemispheres. The principal component and spatial distribution of the second mode for LT4:00 and 10:00 are showed in Fig. 5. The same Fourier analysis depicted by Eq. (7) was utilized to demodulate the amplitudes of annual and semiannual oscillations, as showed in Fig. 6. It was found that annual component has 5-8 times higher oscillation amplitude than semiannual component in the second mode for the both epochs. At dawn time LT 4:00, solar conditions have higher effects on the 
amplitudes of annual and semiannual oscillations in the second mode than at LT 10:00. For example, the amplitudes of annual and semiannual oscillations for LT 4:00 under high solar activity (20002002, 2012-2013) are up to five times of those under low solar activity (2007-2009), while the amplitudes of annual and semiannual oscillations for LT 10:00 during 1999-2010 vary small in quantity ranging from 200 to 300 . At dawn time, the annual and semiannual oscillations have $180^{\circ}$ phase shift, whereas they have no significant phase shift at noon time.
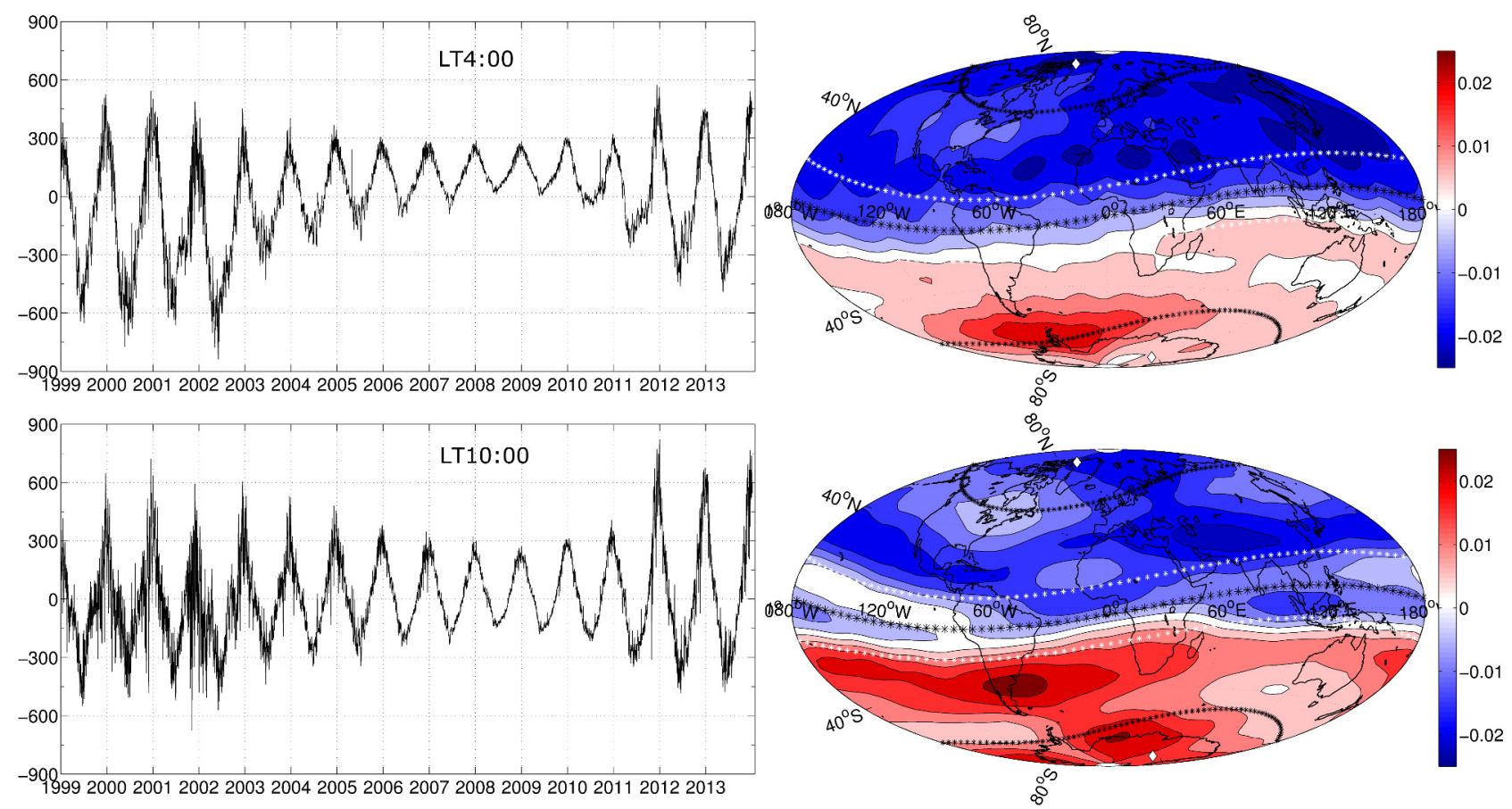

Fig. 5 Temporal and spatial variations in the second mode of the ionosphere of 1999-2013 at local times: LT 4:00 (upper) and LT10:00 (lower).

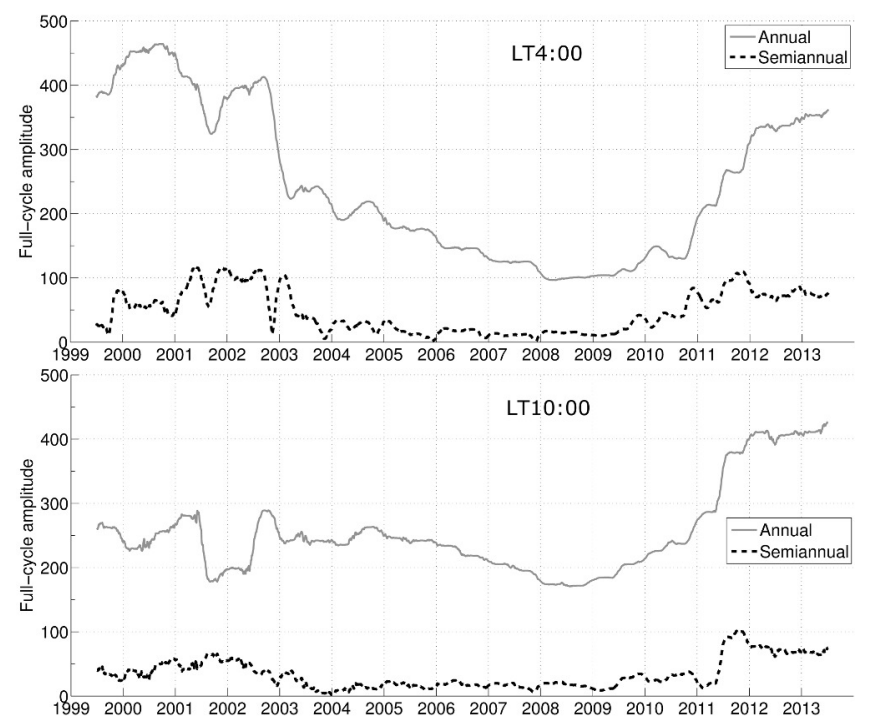

Fig. 6 The amplitudes of annual and semiannual components in the temporal variation of the second mode for local time LT 4:00 and LT 10:00, respectively.

\subsection{Analysis using yearly TEC series}

This section presented temporal variation and spatial structure of the TEC series of every individual year from 1999 to 2013. The twelve TEC dataset of every year that refer to every two hours local 
time are processed separately through the PCA method. It was found that the temporal and spatial variability of the ionosphere vary from year to year with solar activity conditions. This section presented selectively the results of four years that represent four main phases of a solar cycle: solar activity peak (2001), solar activity decreasing (2005), solar activity calm (2008) and solar activity arising (2010). Fig. 7 - Fig. 10 illustrate the spatial variations of the first two modes at the two characteristic epochs LT4:00 and LT10:00 of the selected four years, respectively, and Fig. 11 - Fig. 14 presented the corresponding temporal variations. For Fig. 11 to Fig.14, the x-axis represents the Day of Year (DOY) and the y-axis the amplitude of principal components.

For dawn time LT4:00, spatial variation of the first mode illustrated in Fig. 7 is anticorrelated and asymmetric in Southern and Northern hemispheres. The degree of asymmetry increases as solar activity arises. The maximum variation is distributed to the high ML region in the both Southern and Northern hemispheres. When solar activity becomes active, the area of the maximum variation of Southern hemisphere spread equatorward, and the variation at Pacific Ocean and Atlantic Ocean of Southern hemisphere is higher than that of others. Fig. 11 illustrated the principal component of mode 1 of the decomposition for dawn time of the selected four years. The temporal variations showed obvious annual oscillation. When solar activity is arising (2010) or is peak (2001), winter maximum was clearly observed. Winter maximum becomes weaker or even disappears when solar activity calms down, for example, in 2005 and 2008.

For LT10:00, Fig. 8 showed that the maximum variation of the first mode under the various solar conditions is distributed to the band from $0^{\circ}$ to $\pm 20^{\circ} \mathrm{ML}$, where equatorial anomaly occurs. TEC variation of the first mode decreases as the ML increases in the both hemispheres, while it is asymmetric at the global. The variation is higher in the southern hemisphere, especially for the latitudinal band from southern $15^{\circ}$ to $30^{\circ}$ ML. During most of the solar cycle, TEC variation of the first mode is anticorrelating at geomagnetic polar areas of the both hemispheres, which extend in spatial size with decreasing solar activity. Solar activity has positive effect on TEC variation of mode 1 of middle ML in the both hemispheres. When the solar becomes increasingly active (e.g. in 2010), the spatial structure of the first mode evolves toward that of peak solar activity (e.g. 2001). TEC temporal variations of the first mode for LT10:00 represent a main semiannual variation demodulated by an annual variation, as shown in Fig. 12. Relative rate factors of semiannual and annual variations in the principal component of the first mode vary with different phases of a solar cycle. When solar activity is arising (2010) or at peak (2001), maximal values of TEC variation occurred at October, and they are roughly $20 \%$ higher than the second peak at March. In contrary, when solar activity is decreasing (2005) or under calm condition (2008), the maximal values occurred at March, and they were roughly 20\% higher than the second peak at October.

As to the second, the spatial structure of TEC variation at LT4:00 is significantly different for various solar conditions, as showed in Fig. 9. Under active solar condition of 2001, TEC variation is anticorrelating at Southern and Northern hemispheres, and it is asymmetric with a higher amplitude in northern hemisphere. When solar activity decreases (e.g. 2005), the spatial variation become correlating around the global, and minimal variations distribute at south Indian Ocean area (high $\mathrm{ML}$ ). Under calm solar activity, high ML areas (around $\mathrm{ML60}{ }^{\circ}$ ) of the both hemispheres have high and correlating variations, and the variation of these areas is anticorrelating with that of low and middle ML. As solar activity arises, the variations of high ML of the both hemispheres become anticorrelating, and the areas of higher variations in the both hemispheres extend gradually from high ML areas to the whole hemispheres, respectively. Thus, when solar activity comes to the next peak in 2013, spatial structure of TEC variation becomes into a similar one as 2001. As showed in Fig. 13, TEC temporal variations of the second mode of dawn time represent semiannual variation 
demodulated with annual variation, and the amplitudes are positively correlated with solar conditions.

At LT10:00, spatial variations of the second mode are highly dependent of latitudes and asymmetric between the both hemispheres. TEC variation is higher in two groups of area: one is the latitudinal band from southern $15^{\circ}$ to northern $30^{\circ}-50^{\circ} \mathrm{ML}$, the size of which depends on the phase of solar cycle; the other is the geomagnetic polar areas $\left(>60^{\circ} \mathrm{ML}\right)$ of the both hemispheres. During most of the solar cycle (except 2001), TEC variation is anticorrelating in the two parts separated by ML $15^{\circ}$. TEC temporal variations of the second mode in Fig. 14 show the maximal values during the both equinoxes, and the March-April equinox peak is higher than the September-October one.
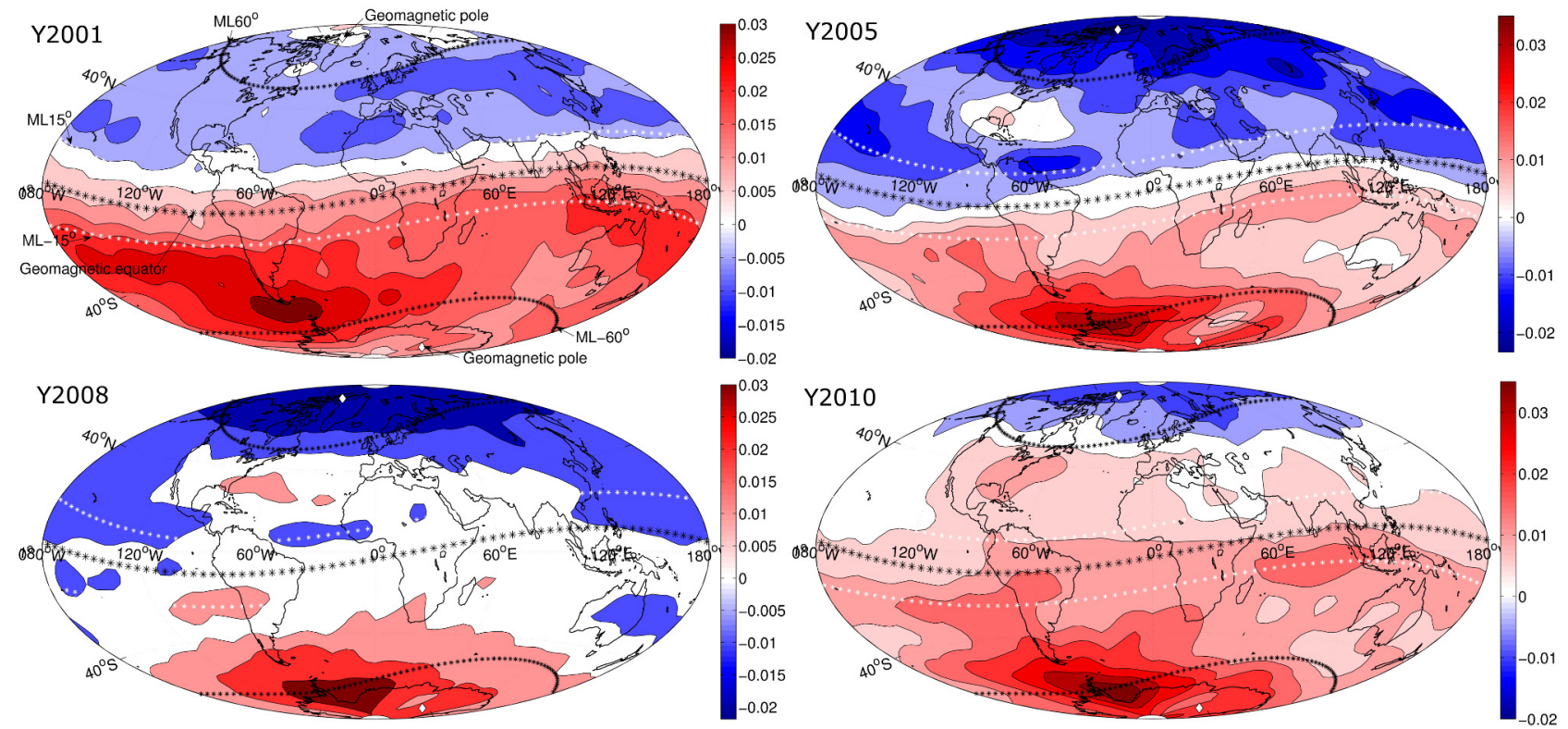

Fig. 7 Spatial variations in the first mode of the ionosphere of the selected four years for local time 4:00. In the four subplots, the colormap scales are unified for comparison.
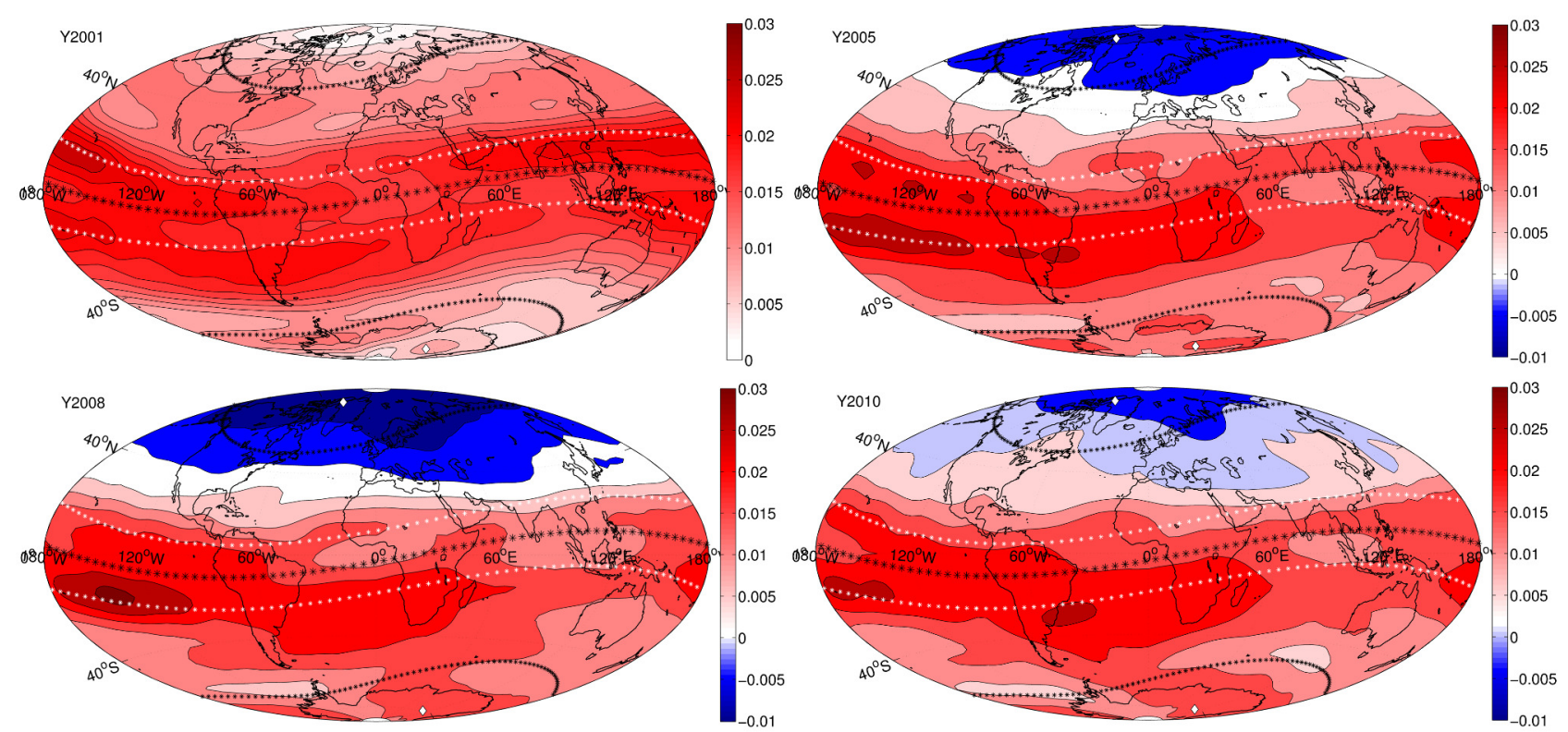

Fig. 8 Spatial variations in the first mode of the ionosphere of the selected four years for local time 10:00. In the four subplots, the colormap scales are unified for comparison. 

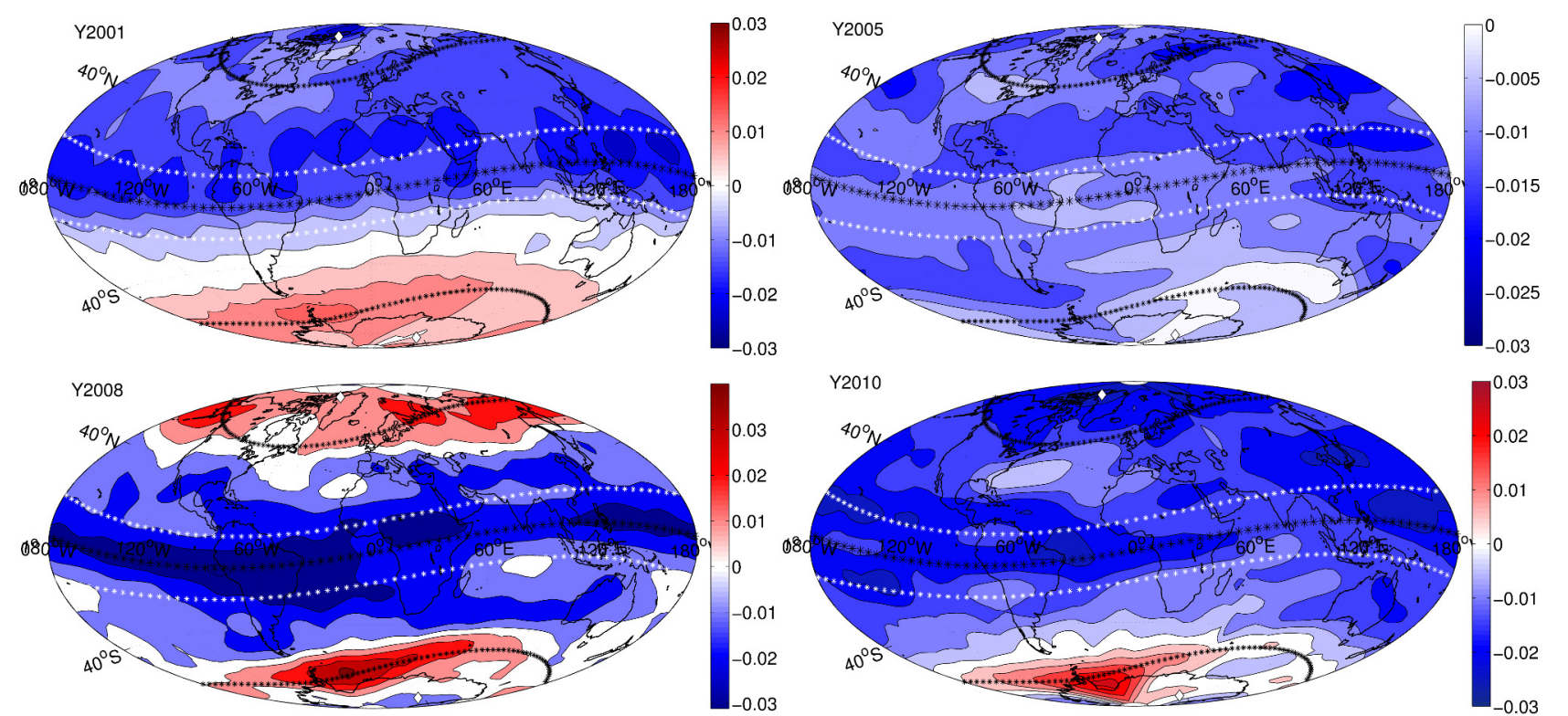

Fig. 9 Spatial variations in the second mode of the ionosphere of the selected four years for local time 4:00. In the four subplots, the colormap scales are unified for comparison.
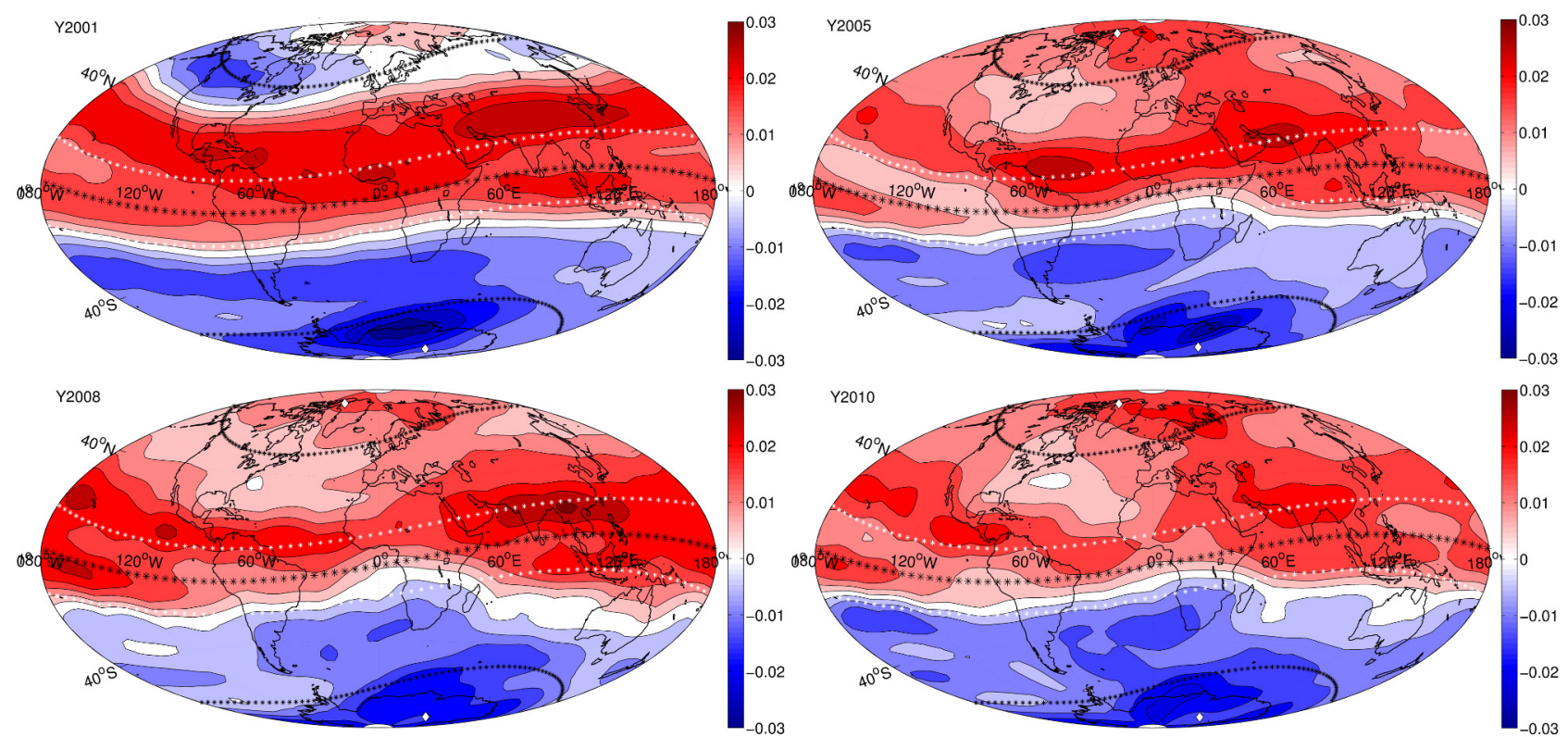

Fig. 10 Spatial variations in the second mode of the ionosphere of the selected four years for local time 10:00. In the four subplots, the colormap scales are unified for comparison. 


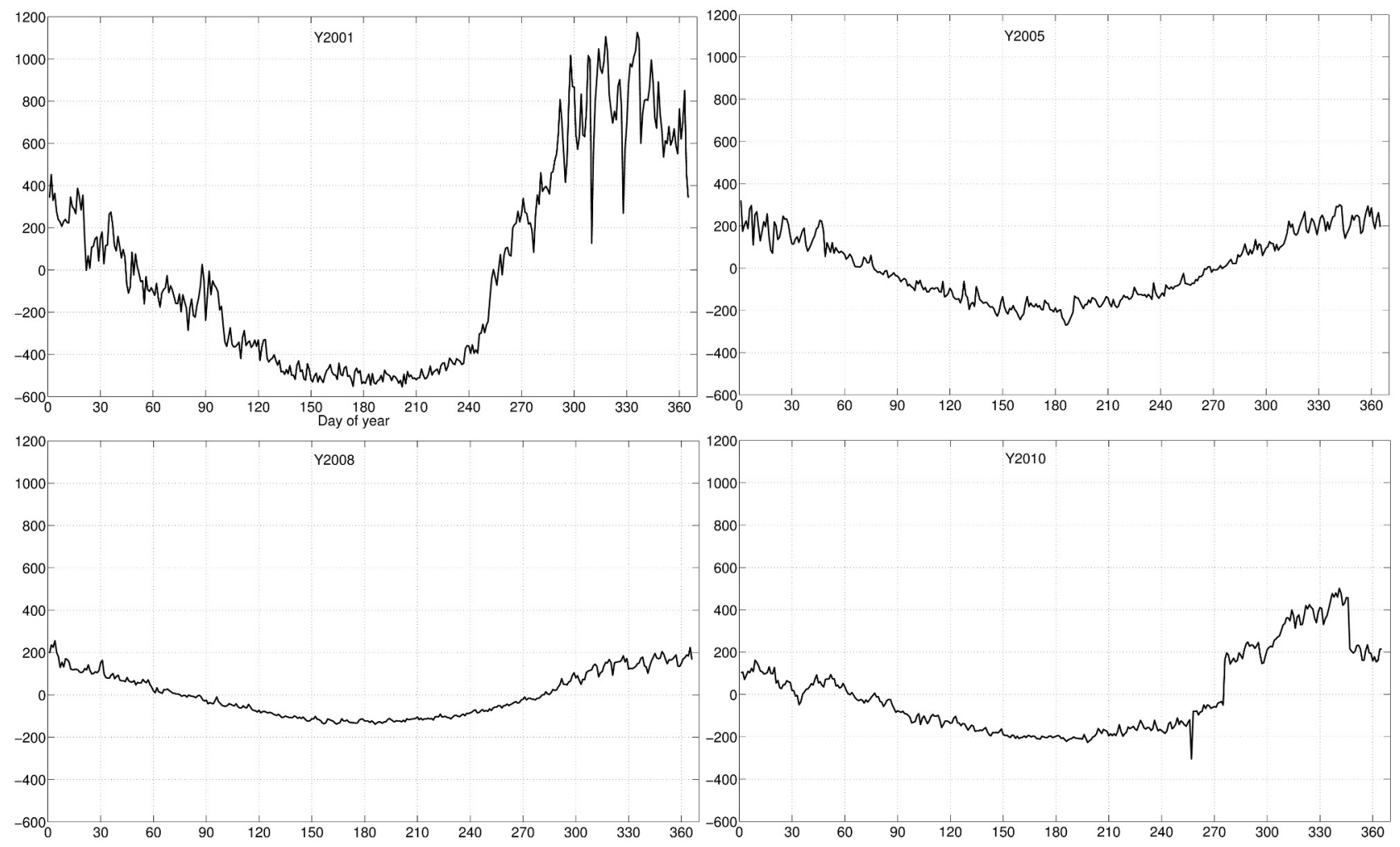

Fig. 11 Temporal variations in the first mode for LT 4:00 of the selected four years.

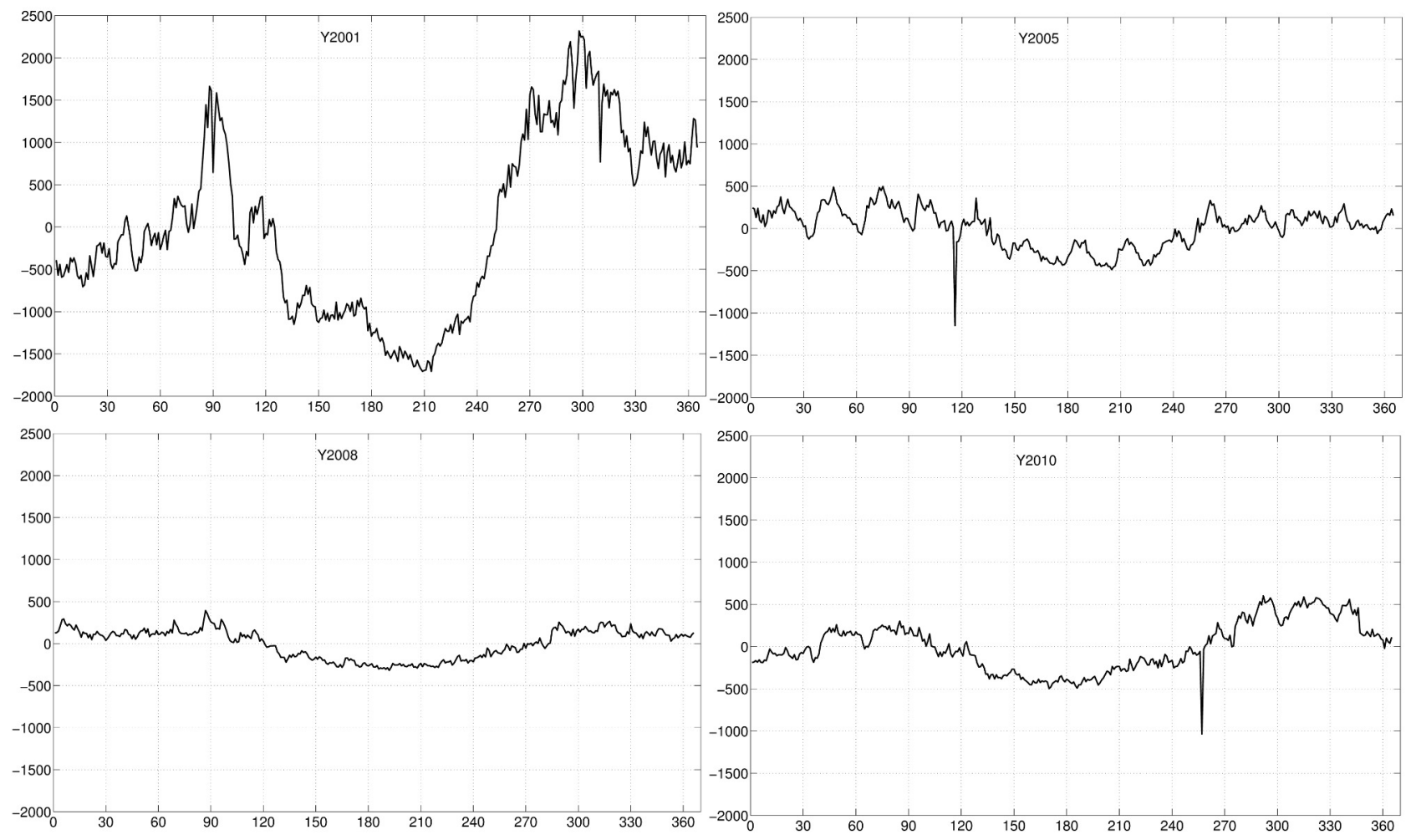

Fig. 12 Temporal variations in the first mode for LT 10:00 of the selected four years. 


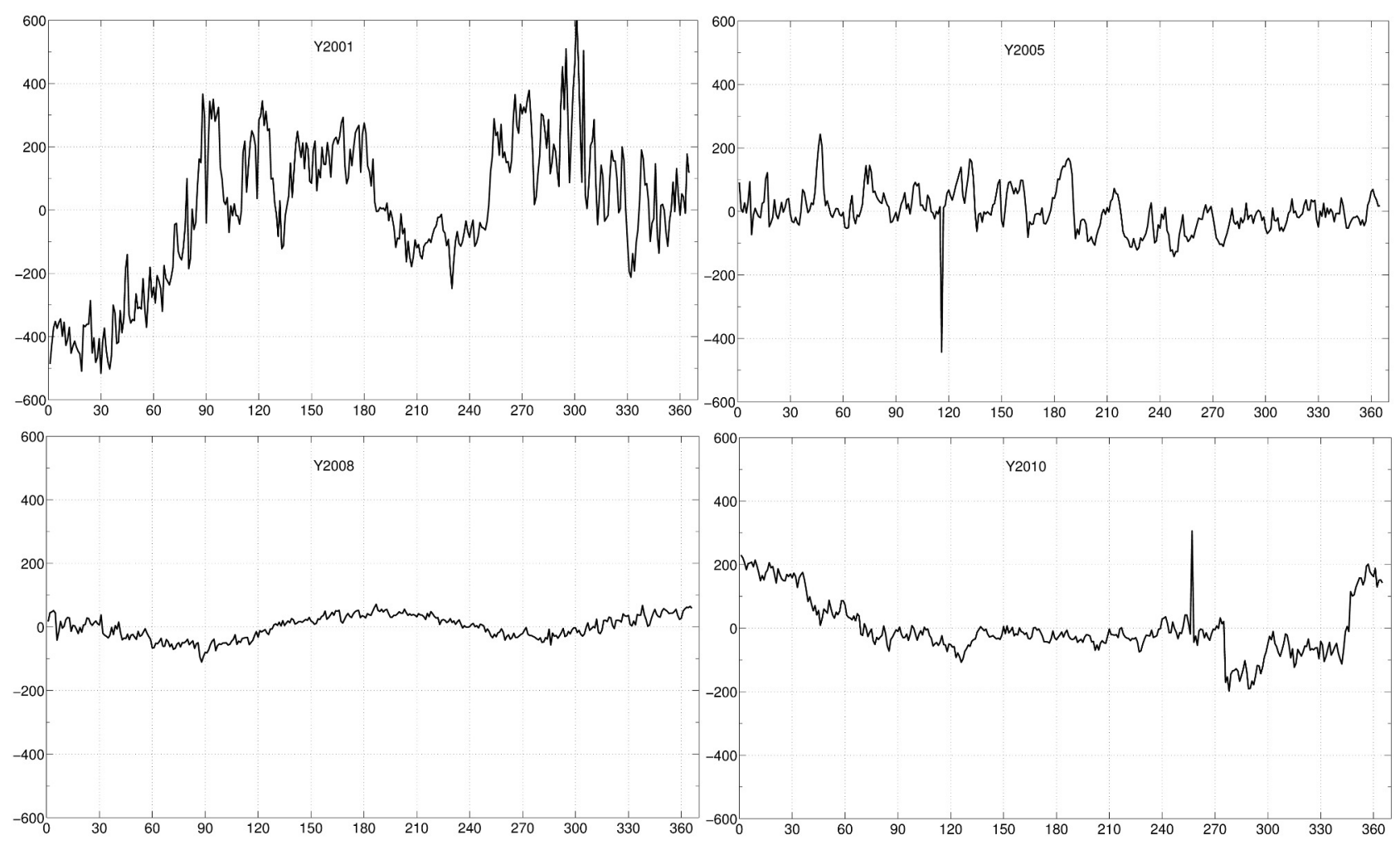

Fig. 13 Temporal variations in the second mode for LT 4:00 of the selected four years.

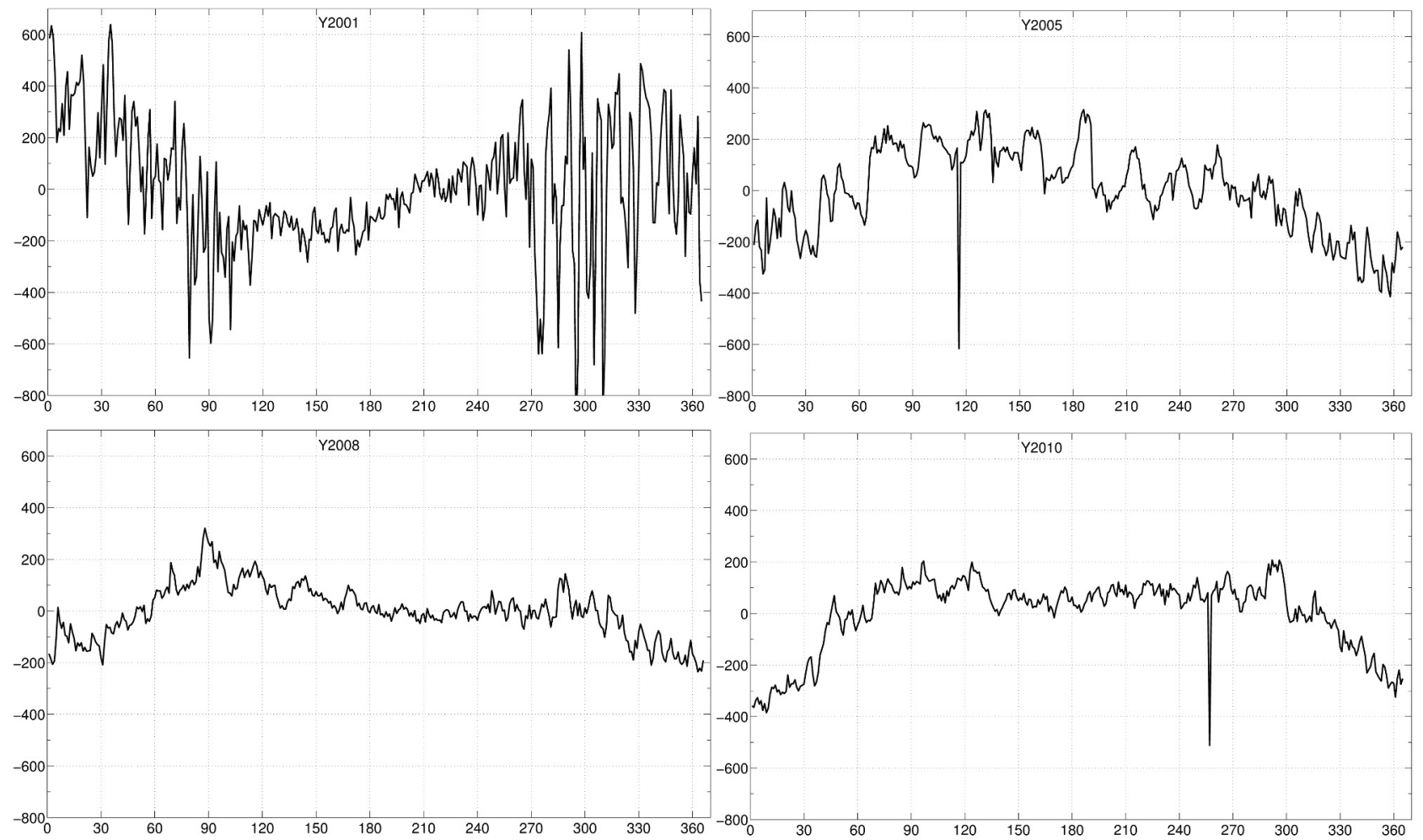

Fig. 14 Temporal variations in the second mode for LT 10:00 of the selected four years.

The first two modes, modulated with both annual and semiannual variations, account for an integrated relative variance of 70\%-90\%, varying with solar conditions and local times. Annual and semiannual components of the first modes are demodulated from the amplitude series using the Fourier analysis method as Eq.(7), and normalized rate factors $R$ of these two variations are 
calculated using the parameters $\left(A_{i}\right)$ in Eq.(7) as: $R_{a}=\frac{A_{1}}{A_{1}+A_{2}}, R_{S}=\frac{A_{2}}{A_{1}+A_{2}}$, where $R_{a}$ and $R_{S}$ are rate factors of annual and semiannual components, respectively. It is obvious that, for each specific section of local time of a specific year, $R_{a}+R_{s}=1$. Fig. 15 illustrates the rate factors of annual and semiannual components in the first two modes at different years and local times. It was found that the variability of daytime is more of compound, and the first two modes of nighttime (from dusk time to dawn time) are virtually dominated by either annual variation or semiannual variation. These factors are also dependent of solar conditions. An exception is that under the active solar conditions (2000-2003), the variability of the earlier nighttime (LT18:00 - 22:00) keeps the similar as that of daytime, and it shows that extremely strong solar irradiance of daytime has remaining effect on the ionosphere even after the sunset.

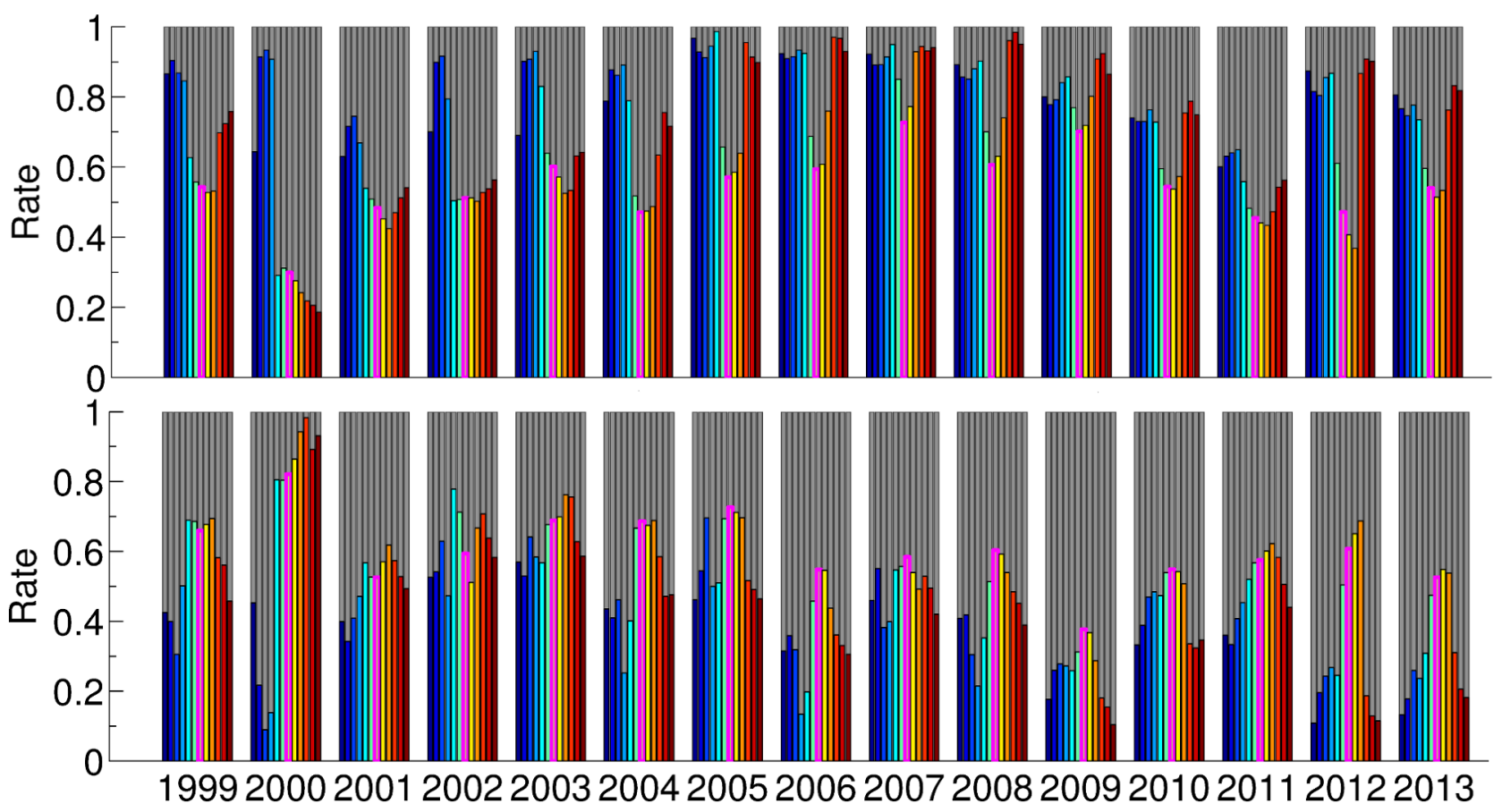

Fig. 15 Rate factors of annual and semiannual components in the first mode (upper) and the second mode (lower) for different years and local times. For each year, twelve bars are related to the twelve reference local times in sequence. The colorful parts indicate the rate factors of annual components $\left(R_{a}\right)$, while the grey parts indicate the rate factors of semiannual components $\left(R_{S}\right)$. The magenta bar corresponds to local time 12:00.

Finally, integrated annual and semiannual variations in the first two modes of different years and local times are calculated as Eq.(8) using these rate factors, and the results are given in Table 2.

$V_{a}=R_{a}^{1} * q_{1}+R_{a}^{2} * q_{2}$

$V_{s}=R_{S}^{1} * q_{1}+R_{S}^{2} * q_{2}$

where $V_{a}$ and $V_{s}$ are the normalized amplitudes of annual and semiannual variations, respectively, $R_{a}^{i}, i=1,2$ are rate factors of annual components in the first and second modes, respectively, $R_{s}^{i}, i=1,2$ are rate factors of semiannual components in the first and second modes, respectively, and $q_{i}, i=1,2$ are eigenvalues of the first and second modes, respectively.

Table 2 presented the normalized amplitudes of annual and semiannual variations of different years and local times, which have been decomposed from the first two modes of PCA. It was found that annual and semiannual variations have different trends through the various phases of a solar cycle. 
Annual variation is more significant under lower solar activity conditions, e.g. during the recent solar minima of 2007-2009, whereas semiannual variation is higher under active solar conditions. Annual variation of night times is much higher than that of daytime. The peak annual variation occurs at dawn time (LT 4:00-6:00), and on average it has 1.5-2 times of the amplitude of annual variation of noon time (LT 12:00-14:00) throughout the various solar conditions. In contrary, semiannual variation of daytime is much higher than that of night time. The peak semiannual variation occurs at afternoon (LT 14:00-16:00) under active solar conditions (1999-2003, 20112013), and at noon time (LT 12:00) under lower solar activity. The amplitude of the peak semiannual variation is 2-3 times higher than that of dawn time. When it is the early stage of active solar condition such as in 2000 and 2011, semiannual variation of LT 14:00-16:00 has the highest amplitude, and the amplitude is even higher than that of annual variation of the same period. When solar activity comes to the peak (e.g. 2001-2002, and 2012-2013), the amplitudes of semiannual variation even decrease. Daytime solar irradiance under active solar conditions has remaining effect on the ionosphere even after the sunset when the solar is of high activity.

Table 2. Normalized amplitudes (\%) of the annual and semiannual variations at the different local times during $1999-2013$

\begin{tabular}{|c|c|c|c|c|c|c|c|c|c|c|c|c|c|}
\hline Year & Local time & 0 & 2 & 4 & 6 & 8 & 10 & 12 & 14 & 16 & 18 & 20 & 22 \\
\hline \multirow{2}{*}{1999} & & 54.58 & 56.39 & .51 & 62.47 & 51.31 & 45.42 & 43.11 & 2.71 & 5.70 & 2.95 & 1.82 & 0.22 \\
\hline & Semiannual & 17.85 & 14.47 & 19.00 & 19.81 & 28.39 & 33.51 & 34.33 & 35.31 & 35.09 & 26.87 & 24.59 & 24.88 \\
\hline \multirow{2}{*}{2000} & Annual & 41.56 & 48.12 & 55.72 & 51.59 & 31.64 & 28.53 & 27.42 & 25.41 & 26.51 & 36.71 & 35.38 & 37.93 \\
\hline & Semiannual & 33.59 & 21.41 & 16.69 & 27.32 & 45.14 & 49.91 & 51.99 & 53.56 & 4.19 & 44.22 & 42.04 & 40.52 \\
\hline \multirow{2}{*}{2001} & Annual & 44.87 & 47.98 & 53.71 & 53.75 & 46.72 & 44.27 & 41.72 & 38.75 & 37.29 & 40.96 & 41.89 & \\
\hline & Semiannual & 33.88 & 27.92 & 24.91 & 31.85 & 39.35 & 42.57 & 44.14 & 46.11 & 47.73 & 42.88 & 39.38 & 37.67 \\
\hline \multirow{2}{*}{2002} & Annual & 48.43 & 58.33 & 64.98 & 56.15 & 45.65 & 43.85 & 42.72 & 41.90 & 43.04 & 45.78 & 43.21 & 43.37 \\
\hline & Semiar & 26.39 & 14.12 & 10.74 & 26.24 & 37.39 & 40.14 & & & & & & \\
\hline \multirow{2}{*}{2003} & Annual & 48.87 & 55.47 & 60.14 & 70.41 & 61.33 & 50.03 & 47.22 & 44.50 & 43.08 & 47.77 & 49.14 & 47.88 \\
\hline & Semiannual & 26.58 & 16.51 & 10.99 & 10.07 & 18.75 & 27.59 & 30.09 & 31.49 & 33.57 & 32.16 & 28.84 & 28.74 \\
\hline \multirow{2}{*}{2004} & Annual & 49.61 & 52.57 & 56.43 & 65.28 & 54.99 & 42.95 & 39.67 & & 42.16 & 49.56 & 51.40 & 48.79 \\
\hline & Semiannual & 24.36 & 17.79 & 14.68 & 16.63 & 26.02 & 35.01 & 38.44 & 37.81 & 35.91 & 30.96 & 26.72 & 27.41 \\
\hline \multirow{2}{*}{2005} & Annual & 56.50 & 56.16 & 61.68 & 72.10 & 68.11 & 46.98 & 45.66 & 46.17 & 0.12 & 2.51 & 0.81 & 55.95 \\
\hline & Semiannual & 13.59 & 11.13 & 8.50 & 8.58 & 10.88 & 23.37 & 28.57 & 27.61 & 25.30 & 17.34 & 16.97 & 17.23 \\
\hline \multirow{2}{*}{2006} & Annual & 54.34 & 55.33 & 61.34 & 70.35 & 63.37 & 47.66 & 43.85 & 44.34 & 49.63 & 64.04 & 64.54 & 57.59 \\
\hline & Semiannual & 16.49 & 13.51 & 12.26 & 13.13 & & & & & 8.72 & & & 16.99 \\
\hline \multirow{2}{*}{2007} & Annual & 59.33 & 58.73 & 62.44 & 73.05 & 74.27 & 60.47 & 52.80 & 53.74 & 62.38 & 69.95 & 68.56 & 63.31 \\
\hline & Semiannual & 12.16 & 11.12 & 12.17 & 10.52 & 9.25 & 18.40 & 24.17 & 22.86 & 5.93 & 11.92 & 11.56 & 11.57 \\
\hline \multirow{2}{*}{2008} & Annual & 57.10 & 56.71 & 59.81 & 68.99 & 68.65 & 53.71 & & & 54.84 & 67.92 & 69.89 & 62.71 \\
\hline & Semiannual & 17.33 & 16.87 & 15.49 & 13.29 & 15.52 & 28.53 & 32.31 & 30.93 & 27.14 & 15.44 & 11.64 & 14.58 \\
\hline \multirow{2}{*}{2009} & Annual & 51.34 & 51.53 & 55.85 & 65.15 & 64.67 & 54.30 & 49.59 & & 52.97 & 62.42 & 64.63 & 56.55 \\
\hline & Semiannual & 21.18 & 20.39 & 19.69 & 17.55 & 18.81 & 25.70 & 28.69 & 28.22 & 26.17 & 19.18 & 14.54 & 18.55 \\
\hline \multirow{2}{*}{2010} & Annual & 51.63 & 54.07 & 57.26 & 62.97 & 58.92 & 49.69 & 45.31 & 44.43 & 46.22 & 52.28 & 54.83 & 52.05 \\
\hline & Semiannual & 27.62 & 27.07 & 26.30 & 25.56 & 29.07 & 35.26 & 37.83 & 38.12 & 37.14 & 32.19 & 27.32 & 27.43 \\
\hline \multirow{2}{*}{2011} & Annual & 47.52 & 49.89 & 53.48 & 56.91 & 51.30 & 45.10 & 42.08 & 40.96 & 41.28 & 44.70 & 46.88 & 46.61 \\
\hline & Semiannual & 38.68 & 36.19 & 34.01 & 34.70 & 41.56 & 46.79 & 48.46 & 49.08 & 48.99 & 45.44 & 40.78 & 40.06 \\
\hline \multirow{2}{*}{2012} & Annual & 51.24 & 53.44 & 57.68 & 66.53 & 59.24 & 44.54 & 38.10 & 35.35 & 37.15 & 46.70 & 52.01 & 50.86 \\
\hline & Semiannual & 24.75 & 21.41 & 19.80 & 17.60 & 23.21 & 32.59 & 36.91 & 39.29 & 39.91 & 33.70 & 27.29 & 26.25 \\
\hline
\end{tabular}




\begin{tabular}{c|l|c|c|c|c|c|c|c|c|c|c|c|c}
\hline \multirow{2}{*}{2013} & Annual & 53.14 & 54.50 & 57.56 & 62.74 & 56.44 & 47.37 & 43.17 & 40.83 & 42.30 & 50.68 & 54.72 & 53.23 \\
\cline { 2 - 11 } & Semiannual & 25.66 & 25.00 & 25.73 & 25.48 & 30.82 & 35.79 & 37.15 & 37.43 & 36.79 & 31.83 & 26.96 & 26.22 \\
\hline
\end{tabular}

\section{Conclusions and outlook}

This study analyzed the spatial and temporal variability of the ionospheric TEC under the various solar conditions. The numerical technique used in this paper is the Principal Component Analysis. The dataset of global TEC maps of 1999-2013, which covers a period of more than a solar cycle, are first reorganized according to local times. The TEC series of different local times are then processed separately through the PCA technique in two time scales: one is the whole 15 years of the period of study; and the other is every individual year. Both spatial structure and temporal variation of ionosphere variability are analyzed under the two time scales. This work presented quantitative outcomes and new observations related to the variability of the global ionosphere, thanks to the twofold diverseness from previous studies: one is the new data representation method; and the other is the long-time dataset that cover various solar conditions during the period of more than a solar cycle. The analyses on the ionosphere variability are summarized as follows.

Firstly, the ionosphere has significantly different temporal and spatial variability for daytime and nighttime. Different from previous studies, which assumed LT 12:00 and 22:00 as characteristic epochs of study, this study presented the ionosphere variability of every two hours local time, and showed that dawn time (e.g. LT4:00) and the later morning (LT10:00-12:00) are more remarkable characteristic epochs for the ionosphere variability. This work showed also that the variability of the ionosphere varies with local time under a diurnal cyclic trend.

Secondly, PCA performs the orthogonal decomposition of the data itself, and it does not force any specific forms of components. This feature provides the PCA technique an ability to freely decompose principal components of minimum dimensions, from which it is possible to observe asymmetries such as semiannual anomaly and seasonal anomaly, for example, in Fig. 11- Fig. 14. These kinds of asymmetry will be removed due to mismodeling in techniques that involve specifically predefined functions, according to the previous works.

Thirdly, the first multiple modes contribute to most of the temporal and spatial variability that vary with solar conditions and local times. The first and second modes account for $80 \%-90 \%$ of the total variance over a solar cycle, and the corresponding spatial and temporal variations are presented in terms of characteristic local times and typical phases of solar cycle. The amplitudes of annual and semiannual oscillations demodulated from the first two modes are quantitatively analyzed. It was found that the ionosphere behaves with evidently different patterns of the variability for daytime and nighttime, which were presented in this work. An exception is that, under active solar conditions, extremely strong solar irradiance of daytime has remaining effect on the variability of the ionosphere even after the sunset (e.g. from LT 18:00 to LT 22:00).

Finally, a global TEC climatological model will be constructed in further work using the results of applying the PCA technique to the long-time TEC GIMs, thanks to the highly convergent representation of PCA for the ionosphere variability.

\section{Acknowledgements}

The CODE GIM dataset used in this study has been downloaded from CODE's data archive server (ftp://ftp.unibe.ch/aiub/CODE), and the solar and geomagnetic indices have been downloaded from the national geophysical data center (ftp://ftp.ngdc.noaa.gov/STP/). This work was supported in part 
by the Finnish Centre of Excellence in Laser Scanning Research (CoE-LaSR, grant number 272195) of Academy of Finland.

\section{References}

1. Garcia-Rigo A, Monte E, Hernandez-Pajares M, Juan JM, Sanz J, et al. (2011) Global prediction of the vertical total electron content of the ionosphere based on GPS data. Radio Science 46 (RS0D25). http://dx.doi. org/10.1029/2010RS004643.

2. Garner TW, Gaussiran T, Tolman BW, Harris RB, Calfas RS, et al. (2008) Total electron content measurements in ionospheric physics. Adv Space Res 42(4): 720-726. doi:10.1016/j.asr.2008.02.025.

3. Dikpati M (2008) Predicting cycle 24 using various dynamo-based tools. Ann Geophys 26: 259 - 267, 2008. doi:10.5194/angeo-26-259-2008.

4. Gulyaeva TL, Arikan F, Stanislawska I, Poustovalova LV (2012) Symmetry and asymmetry of ionospheric weather at magnetic conjugate points for two midlatitude observatories. Adv in Space Res 52(10): 1837-1844. DOI: 10.1016/j.asr.2012.09.038.

5. National Research Council (2003) The Sun to the Earth and beyond: A decadal research strategy in solar and space physics. Natl. Acad. Press, Washington, D.C.

6. National Space Weather Strategic Plan (1995) Official Fed. Coord. for Meteorol. Services, NOAA, Silver Spring, Md.

7. Hernández-Pajares M, Juan JM, Sanz J, Aragón-Àngel A (2012) Propagation of medium scale traveling ionospheric disturbances at different latitudes and solar cycle conditions. Radio Sci 47, RS0K05, doi:10.1029/2011RS004951.

8. Pesnell WD (2012) Solar cycle predictions. Sol Phys 281(1): 507-532, doi: 10.1007/s11207012-9997-5

9. Sardon E, Rius A, Zarraoa N (1994) Estimation of the transmitter and receiver differential biases and the ionospheric total electron content from Global Positioning System observations, Radio Sci 29(3): 577-586.

10. Afraimovich EL, Astafyeva EI, Oinats AV, Yasukevich YV, Zhivetiev IV (2008) Global electron content: A new conception to track solar activity. Ann Geophys, 26: 335-344, doi:10.5194/angeo-26-335-2008.

11. Hernández-Pajares M, Juan JM, Sanz J, Orus R, Garcia-Rigo A, et al. (2009) The IGS VTEC maps: a reliable source of ionospheric information since 1998, J Geod 83:263-275, DOI 10.1007/s00190-008-0266-1

12. Wu Y, Jin S, Wang Z, Liu J (2010) Cycle-slip detection using multi-frequency GPS carrier phase observations: a simulation study. Adv Space Res, 46:144-149.

13. Scharroo R, Smith WHF (2010) A global positioning system-based climatology for the total electron content in the ionosphere, J Geophys Res 115, A10318, doi:10.1029/2009JA014719.

14. Schaer S (1999) Mapping and predicting the earth's ionosphere using the global positioning system. Doctor Thesis, The University of Bern. Available: http://www.sgc.ethz.ch/sgcvolumes/sgk-59.pdf. Accessed 21 July 2014.

15. Liu J, Chen R, An J, Wang Z, Hyyppä J (2014) Spherical cap harmonic analysis of the Arctic ionospheric TEC for one solar cycle. J Geophys Res Space Physics, 119, doi: 10.1002/2013JA019501.

16. Lean JL, Meier RR, Picone JM, Emmert JT (2011) Ionospheric total electron content: Global and hemispheric climatology. J Geophys Res, 116, A10318, doi:10.1029/2011JA016567.

17. Liu L, Wan W, Ning B, Zhang ML (2009) Climatology of the mean total electron content derived from GPS global ionospheric maps. J Geophys Res, 114, A06308, doi:10.1029/2009JA014244.

18. Gulyaeva TL, Arikan F, Hernandez-Pajares M, Stanislawska I (2013) GIM-TEC adaptive ionospheric weather assessment and forecast system, Journal of Atmospheric and Solar- 
Terrestrial Physics, 102: 329-340, 2013. http://dx.doi.org/10.1016/j.jastp.2013.06.011.

19. Ivanov-Kholodny GS, Mikhailov AV (1986) The prediction of ionospheric conditions. ISBN 390-277-2143-2.

20. Zolesi B, Cander LR (2014) Ionospheric prediction and forecasting, Springer Geophysics Series XII

21. Pietrella M, Perrone L, Fontana G, Romano V, Malagnini A, et al. (2009) Oblique-incidence ionospheric soundings over Central Europe and their application for testing now casting and long term prediction models. Adv Space Res43(11): 1611-1620.

22. Bilitza D, McKinnell LA, Reinisch B, Fuller-Rowell T (2011) The international reference ionosphere today and in the future. J Geod, 85:909-920, DOI 10.1007/s00190-010-0427-x.

23. Jakowski N, Hoque M, Mayer MC (2011) A new global TEC model for estimating transionospheric radio wave propagation errors, J Geod 85(12): 965-974, 2011. http://dx.doi.org/10.1007/s00190-011-0455-1

24. Mannucci AJ, Wilson BD, Yuan DN, Ho CH, Lindqwister UJ, et al. (1998) A global mapping technique for GPS-derived ionospheric total electron content measurements. Radio Sci 33(3): 565-582, doi:10.1029/97RS02707.

25. Hernández-Pajares M, Aragón-Ángel À, Defraigne P, Bergeot N, Prieto-Cerdeira R, et al. (2014) Distribution and mitigation of higher-order ionospheric effects on precise GNSS processing. J Geophys Res Solid Earth, 119: 3823-3837, doi:10.1002/2013JB010568.

26. Liu J, Chen R, Wang Z, Zhang H (2011) Spherical cap harmonic model for mapping and predicting regional TEC. GPS Solut 15: 109-119.

27. Haines GV (1985) Spherical cap harmonic analysis. J Geophys Res 90(B3): 2583-2591

28. Zahra B, Terkildsen M, Neudegg D (2010) Regional GPS-based ionospheric TEC model over Australia using Spherical Cap Harmonic Analysis. The 38th COSPAR Scientific Assembly, Bremen, Germany, 18-15 July 2010.

29. Ghoddousi-Fard R, Héroux P, Danskin D, Boteler D (2011) Developing a GPS TEC mapping service over Canada. Space Weather 9, S06D11, doi:10.1029/2010SW000621.

30. Liu J, Wang Z, Wang H, Zhang H (2008) Modeling regional ionosphere using GPS measurements over China by spherical cap harmonic analysis methodology. Geomat and Inf Sci of Wuhan University 33(8): 792-795.

31. Liu J, Chen R, Kuusniemi H, Wang Z, Zhang H, et al. (2010) A preliminary study on mapping the regional ionospheric TEC using a spherical cap harmonic model in high latitudes and the Arctic region, Journal of Global Positioning Systems. 9(1): 22-32.

32. Wilson BD, Mannucci AJ, Edwards CD (1995) Subdaily northern hemisphere ionospheric maps using an extensive network of GPS receivers. Radio Sci 30: 639-648.

33. Komjathy A (1997) Global ionospheric total electron content mapping using the global positioning system, Doctor Thesis, The University of New Brunswick.

34. Liu J, Wang Z, Zhang H, Zhu W (2008) Comparison and consistency research of regional ionospheric TEC models based on GPS measurements. Geomat and Inf Sci of Wuhan University 33(5): 479-483.

35. Schaer S, Gurtner W, Feltens J, Feltens J (1998) IONEX: The IONosphere map Exchange format version 1. Available: ftp://cddis.gsfc.nasa.gov/reports/formats/ionex1.pdf. Accessed 21 July 2014.

36. Lean JL, Emmert JT, Picone JM, Meier RR (2011) Global and regional trends in ionospheric total electron content. J Geophys Res 116, A00H04, doi:10.1029/2010JA016378.

37. Rishbeth H, Garriott OK (1969) Introduction to ionospheric physics. Volume 14 of International geophysics series, Academic Press, New York and London.

38. Omlin M, Reichert P (1999) A comparison of techniques for the estimation of model prediction uncertainty. Ecological Modelling 115: 45-59.

39. Strangeways HJ, Kutiev I, Cander LjR, Kouris S, Gherm V, et al. (2009) Near-earth space 
plasma modeling and forecasting. Ann Geophys, 52(3): 255-271.

40. Friis-Christensen E, Lassen K (1991) Length of the solar cycle: An indicator of solar activity closely associated with climate, Science, New Series, 254: 698-700.

41. Jingbin Liu, R. Chen, J. An, Z. Wang, and J. Hyyppa, Long-term prediction of the Arctic ionospheric TEC based on time-varying periodograms, PLOS ONE 9(11): e111497. doi:10.1371/journal.pone.0111497.

42. Yu. S. Maslennikova and V. V. Bochkarev (2014), Principal Component Analysis of Global Maps of the Total Electronic Content, Geomagnetism and Aeronomy, 54(2), pp. 216-223, DOI: $10.1134 / \mathrm{S} 0016793214020133$.

43. Natali, M. P. and Meza, A. (2010), Annual and semiannual VTEC effects at low solar activity based on GPS observations at different geomagnetic latitudes, J. Geophys. Res., 115, D18106, doi:10.1029/2010JD014267.

44. Natali, M. P. and Meza, A. (2011), Annual and semiannual variations of vertical total electron content during high solar activity based on GPS observations, Ann. Geophys., 29, 865-873, 2011, doi:10.5194/angeo-29-865-2011. 